\title{
Lipid-related genetic polymorphisms significantly modulate the association between lipids and disability progression in multiple sclerosis
}

\author{
Yan Zhang, ${ }^{1}$ Yuan Zhou ${ }^{1}$ Ingrid A F van der Mei, ${ }^{1}$ Steve Simpson, ${ }^{1,2}$ \\ Anne-Louise Ponsonby, ${ }^{3}$ Robyn M Lucas, ${ }^{4}$ Prudence Tettey, ${ }^{1,5}$ Jac Charlesworth, \\ Karam Kostner, ${ }^{6}$ Bruce V Taylor, ${ }^{1}$ Ausimmune/AusLong Investigators Group
}

\begin{abstract}
${ }^{1}$ Menzies Institute for Medical Research, University of Tasmania, Hobart, Tasmania, Australia

${ }^{2}$ Melbourne School of Population and Global Health, The University of Melbourne, Melbourne, Victoria, Australia ${ }^{3}$ Murdoch Children's Research Institute, The University of Melbourne, Melbourne, Australia ${ }^{4}$ National Centre for Epidemiology and Population Health, Research School of Population Health, College of Medicine, Biology and Environment, Australian National University, Canberra, Australian Capital Territory, Australia

${ }^{5}$ School of Public Health, University of Ghana, Accra, Ghana

${ }^{6}$ Mater Hospital, University of Queensland, Queensland, Australia
\end{abstract}

Correspondence to Professor Bruce V Taylor, Menzies Institute for Medica Research, University of Tasmania, Hobart, TAS 7000 Australia; bruce.taylor@utas. edu.au

Received 24 October 2018 Revised 14 December 2018 Accepted 24 December 2018 Published Online First 19 February 2019

Check for updates

(C) Author(s) (or their employer(s)) 2019. No commercial re-use. See rights and permissions. Published by BMJ.

To cite: Zhang Y, Zhou Y, van der Mei IAF, et al. J Neurol Neurosurg Psychiatry

2019:90:636-641.

\section{ABSTRACT \\ Objective To investigate whether lipid-related or body mass index (BMI)-related common genetic polymorphisms modulate the associations between serum lipid levels, BMI and disability progression in multiple sclerosis (MS).}

Methods The association between disability progression (annualised Expanded Disability Status Scale (EDSS) change over 5 years, $\triangle$ EDSS) and lipid-related or BMI-related genetic polymorphisms was evaluated in a longitudinal cohort $(n=184)$, diagnosed with MS. We constructed a cumulative genetic risk score (CGRS) of associated polymorphisms $(p<0.05)$ and examined the interactions between the CGRS and lipid levels (measured at baseline) in predicting $\triangle E D S S$. All analyses were conducted using linear regression.

Results Five lipid polymorphisms (rs2013208, rs9488822, rs 17173637, rs10401969 and rs2277862) and one BMI polymorphism (rs2033529) were nominally associated with $\triangle E D S S$. The constructed lipid CGRS showed a significant, dose-dependent association with $\triangle E D S S\left(p_{\text {trend }}=1.4 \times 10^{-6}\right)$, such that participants having $\geq 6$ risk alleles progressed 0.38 EDSS points per year faster compared with those having $\leq 3$. This CGRS model explained $16 \%$ of the variance in $\triangle E D S S$. We also found significant interactions between the CGRS and lipid levels in modulating $\triangle E D S S$, including high-density lipoprotein (HDL; $\left.p_{\text {interaction }}=0.005\right)$ and total cholesterol:high-density lipoprotein ratio (TC:HDL; $\mathrm{p}_{\text {interation }}=0.030$ ). The combined model (combination of CGRS and the lipid parameter) explained $26 \%$ of the disability variance for HDL and $27 \%$ for TC:HDL.

Interpretation In this prospective cohort study, both lipid levels and lipid-related polymorphisms individually and jointly were associated with significantly increased disability progression in MS. These results indicate that these polymorphisms and tagged genes might be potential points of intervention to moderate disability progression.

\section{INTRODUCTION}

Multiple sclerosis (MS) is a clinically and pathologically heterogeneous disease of complex aetiology. Like many other chronic debilitating diseases, there is no cure for MS. Hence, a better understanding of the risk factors associated with clinical progression is vital. Compared with the dramatic progress in identifying the genetic and environmental factors associated with MS onset, ${ }^{1}{ }^{2}$ there has been less success with the drivers of MS progression. Although a number of environmental/behavioural determinants of clinical course have been identified, ${ }^{3-5}$ there has been less success with genetic determinants of clinical progression. ${ }^{6}$

The approaches used in detecting genetic factors associated with MS onset-candidate-gene studies, linkage studies or genome-wide association studies (GWASs) - are often cross-sectional or case-control in design, allowing large sample sizes but precluding robust evaluation of clinical progression. Therefore, one method by which the genetic factors that modulate MS clinical course could be elucidated is by studying the genetic loci identified in GWAS that modulate environmental or personal factors associated with MS progression in longitudinal MS clinical cohorts where these factors have been assessed prospectively.

Several studies, including our own work, have shown that serum lipid levels and body mass index (BMI) have significant associations with disability level and disability progression among people with MS. Serum lipids associated both positively and negatively with MS progression include total cholesterol (TC), high-density lipoprotein (HDL), low-density lipoprotein (LDL) and triglycerides. In a study of 8983 participants, higher LDL and lower HDL were associated with greater ambulatory disability. ${ }^{7}$ In a study of 178 participants, higher TC:HDL ratio was associated with higher Expanded Disability Status Scale (EDSS) score. ${ }^{8}$ Our own study with 178 participants showed that higher TC was independently associated with higher EDSS. ${ }^{3}$ A case-control study also demonstrated that TC and LDL were positively correlated with EDSS. ${ }^{9}$ Results for BMI are mixed, however. In our own study, higher BMI was independently associated with higher EDSS, ${ }^{3}$ whereas two other studies failed to find any associations. ${ }^{10} 11$

For disability progression, a prospective study of 492 participants followed for an average of 2.2 years found that higher TC, LDL, triglycerides and TC:HDL ratio were associated with worsening in clinical disability. ${ }^{5}$ Our own study of 279 participants showed that higher TC:HDL ratio, non-HDL level and BMI were associated with faster disability progresssion. ${ }^{4}$ In another of our studies, including 
178 participants, higher TC:HDL ratio was associated with a higher annual change in disability. ${ }^{3}$

However, the mechanisms by which serum lipid level or BMI may influence MS disability are not yet established. A better understanding of the interplay by which related genetic factors impact on disability progression could facilitate the identification of predictive biomarkers of disability and indicate potential intervention targets. In this study, we examined whether known lipid-related or BMI-related genetic polymorphisms were associated with disability progression, and/or whether they exerted their effects, in part by affecting the association between serum lipid levels, BMI and disability progression.

\section{SUBJECTS AND METHODS \\ Study design}

The Ausimmune Longitudinal (AusLong) Study is a clinical cohort study built on the original Ausimmune case-control study, seeking to identify risk factors for the onset and early progression of MS. Since 2009, 279 of the original first clinical demyelinating event case participants have been followed prospectively in the AusLong study with $84.6 \%$ retention at 5 years. By the 5-year review time point, 207 participants had been diagnosed with relapse-onset or progressive-onset MS, with 186 participants having both an EDSS recorded at baseline and at the 5-year review. Among those 186 participants, 184 had genomewide genotyping undertaken and constituted the cohort analysed here.

\section{Literature review of lipid-related GWAS and BMI-related GWAS}

To include as comprehensive a list of genetic loci that predicted lipids and BMI, we searched for lipid-related and BMI-related GWAS systematically in three academic databases (PubMed, Embase and ScienceDirect). We only included GWASs that specifically identified genome-wide significant loci that impacted one or more aspects of the lipid profile and BMI in human subjects and were written in English (for more detailed methods for searching related GWAS, see online supplemental methods). Flowcharts of the review process are shown in online supplementary figure 1 (for lipid-related GWAS search) and online supplementary figure 2 (for BMI-related GWAS search).

The systematic search for lipid-related GWAS yielded 351 studies and for BMI, 199 studies. After removing duplicates and studies that did not meet the inclusion criteria, three lipid-related GWASs ${ }^{12-14}$ and one BMI-related GWAS ${ }^{15}$ were included. For the three lipid-related GWASs, we combined the significant single-nucleotide polymorphisms (SNPs) together, yielding 162 lipid SNPs (see online supplementary table 1). Seventy-four SNPs were HDL related, 61 were LDL related, 74 were TC related and 40 were triglycerides related. Of these, 151 were genotyped in our study, while for the remaining 11 SNPs, a nearest proxy SNP was identified (online supplementary table 3). For the meta-analysis of BMI-related GWAS, 60 out of the 97 SNPs (online supplementary table 2) were directly genotyped in our study, while for the remaining 37 SNPs, a nearest proxy SNP was identified (online supplementary table 3 ).

\section{Measures}

Serum and BMI measurements

Non-fasting serum samples, which are representative of the usual metabolic state, ${ }^{16}$ were collected at baseline and stored at $-80^{\circ} \mathrm{C}$ until use. TC and triglycerides were measured using enzymatic colourimetry (Wako Chemicals, Richmond, Virginia,
USA). HDL was measured using precipitation and enzymatic assay (Wako Chemicals). LDL was measured by direct assay using enzymatic colourimetry (Wako Chemicals). Non-HDL levels were calculated by subtracting HDL cholesterol from the total cholesterol. Baseline serum 25-OH-D was measured with a commercially available radioimmunoassay (DiaSorin, Stillwater, Minnesota, USA). Lipid profiles were measured again at the 5 -year review using the same methods as baseline.

BMI was calculated by the ratio of weight $(\mathrm{kg})$ over the square of height $(\mathrm{m})$ measured at baseline and the 5-year review.

\section{Genotyping}

DNA samples were genotyped using the Illumina Human Exome BeadChip (Illumina Human Exome-12 v.1.2 array). The BeadChip includes $\sim 244000$ exome SNPs with an additional $\sim 87000$ MS-relevant variants added as a customised component. Quality control of the data was conducted as described previously. $^{17}$

\section{Disability progression measurement}

Disability level was assessed by a study neurologist using the EDSS for which they were certified by Neurostatus. For relapseonset MS, the baseline EDSS was assumed to be zero on the day before demyelinating symptom onset. For progressive-onset MS, the baseline EDSS measured when they entered the study was used. Thus, annualised disability progression was calculated as the 5-year EDSS change divided by the duration between the day prior to the episode that brought the participants into the study and the 5-year review for relapse-onset MS (or the duration between the baseline and 5-year reviews for progressive-onset MS). This was then expressed as an annualised change in EDSS $(\Delta \mathrm{EDSS})$.

\section{Statistical analysis}

Linear regression was used to assess associations between lipid-related or BMI-related SNPs and $\triangle$ EDSS. Multivariable models were adjusted for age, sex, study site and whether participants were having a relapse at the 5-year EDSS assessment. Further adjustment of all models for BMI, 25-OH-D and immunotherapy use did not significantly alter outcomes (data not shown). Bonferroni correction was used to adjust for multiple comparisons. ${ }^{18}$ To assess potential type I error, 50000 permutation analyses were conducted as described previously ${ }^{19}$ by randomly reallocating the genotypes in the samples in proportion to that of the original sample's genotype frequencies, and re-running the analyses. The proportion of the 50000 simulated estimates that were significantly greater than that found in the as-measured analyses denoted the significance.

A cumulative genetic risk score (CGRS) ${ }^{19}{ }^{20}$ was constructed to examine the joint genetic effects by combining the nominally associated SNPs $(p<0.05)$ from the association between lipid-related SNPs and $\triangle$ EDSS. For consistency, the minor allele of rs2013208, rs17173637 and rs10401969 was set as the reference allele so that the directional effects of the five lipid-related SNPs on $\triangle$ EDSS were consistent.

The interactions between baseline lipid variables (including HDL, LDL, TC, triglycerides, TC:HDL ratio and non-HDL) and the CGRS generated from the five lipid-related SNPs for predicting $\triangle$ EDSS were assessed using a test of the coefficient of a product term formed from the covariates involved. The interaction analyses were adjusted for the same factors and further adjusted for 25-OH-D. The serum 25-OH-D at baseline did not alter the association between the lipid CGRS and $\triangle$ EDSS nor the 


\begin{tabular}{|c|c|}
\hline Characteristics & $\mathrm{n} / \mathrm{N}(\%)$ \\
\hline Female sex & $149 / 184(81.0 \%)$ \\
\hline Progressive-onset MS & $12 / 184(6.5 \%)$ \\
\hline Relapse within preceding 30 days at 5 -year review & $20 / 184(10.9 \%)$ \\
\hline Statin use at baseline & $3 / 184(1.6 \%)$ \\
\hline \multirow[t]{2}{*}{ MS-specific immunomodulatory therapy use during study } & 136/184 (73.9\%) \\
\hline & Mean (SD; range) \\
\hline Age (years) & $38.46(9.56 ; 18-58)$ \\
\hline EDSS at 5th-year review & $2.09(1.66 ; 0-8)$ \\
\hline \multirow[t]{2}{*}{ Annualised change in EDSS } & $0.29(0.24 ;-0.31$ to 1.17$)$ \\
\hline & Median (IQR) \\
\hline MS duration from symptom onset at baseline (years) & $0.75(0.39-1.73)$ \\
\hline $\mathrm{TC}(\mathrm{mmol} / \mathrm{L})$ & $5.23(4.56-6.06)$ \\
\hline $\mathrm{HDL}(\mathrm{mmol} / \mathrm{L})$ & $1.61(1.40-1.86)$ \\
\hline LDL (mmol/L) & $2.85(2.38-3.42)$ \\
\hline Triglyceride (mmol/L) & $1.39(1.01-2.00)$ \\
\hline TC:HDL ratio & $3.24(2.77-3.85)$ \\
\hline Non-HDL (mmol/L) & $3.52(2.93-4.48)$ \\
\hline Body mass index $\left(\mathrm{kg} / \mathrm{m}^{2}\right)$ & $26.23(23.11-30.35)$ \\
\hline
\end{tabular}

EDSS, Expanded Disability Status Scale; HDL, high-density lipoprotein; LDL, low-density lipoprotein; MS, multiple sclerosis; TC, total cholesterol.

association between the lipid CGRS and lipid levels in predicting $\triangle$ EDSS.

We then calculated $\mathrm{R}^{2}$ values for the fraction of the variance in disability explained by the regression model for the lipid variable only (environment), the CGRS only (genes) and the combination of the lipid variable and the CGRS (gene plus environment). The changes in lipid variables and BMI during the 5 years were also tested. We then assessed the association between changes in lipid variables and $\triangle E D S S$, and the interactions between changes in lipid variables and the CGRS in predicting $\triangle$ EDSS. Statistical analyses were conducted in Stata/SE V.14.0 (StataCorp LP, College Station, Texas, USA).

\section{RESULTS}

\section{Participant characteristics}

The 184 participants were $81.0 \%$ women, 6.5\% progressive-onset, of mean age of $38.46 \pm 9.56$ years. The mean EDSS of the participants at 5-year review was $2.09 \pm 1.66$ and mean annualised disability progression was $+0.29 \pm 0.24$ EDSS points. The median BMI was $26.23 \mathrm{~kg} / \mathrm{m}^{2}$ and the median TC was 5.23 $\mathrm{mmol} / \mathrm{L}$. Other cohort characteristics are displayed in table 1.

\section{Association between lipid-related SNPs and $\triangle$ EDSS}

Five of the 162 lipid-related SNPs were nominally $(\mathrm{p}<0.05)$ associated with $\triangle E D S S$, though none remained significant after multiple testing correction (table 2). Of these five SNPs, three (rs2013208, rs9488822 and rs2277862) showed a clear dose dependency, while for the two others (rs17173637 and rs10401969) small cell size precluded evaluation of dose dependency. The permutation simulation analysis for the five significant SNPs showed that the permutation $\mathrm{p}$ values were

Table 2 Five lipid-related SNPs nominally associated with annualised change in EDSS and their cumulative effect on annualised change in EDSS

\begin{tabular}{|c|c|c|c|c|c|}
\hline \multirow[b]{2}{*}{ SNP } & \multirow[b]{2}{*}{ Nearest gene } & \multirow{2}{*}{$\begin{array}{l}\text { Related lipid profile(s) in GWAS (directional } \\
\text { effect with corresponding lipid profile)* }\end{array}$} & \multirow[b]{2}{*}{ Genotype } & \multicolumn{2}{|c|}{$\Delta$ EDSS } \\
\hline & & & & $n$ & $\boldsymbol{\beta}(95 \% \mathrm{Cl}) \dagger$ \\
\hline \multirow[t]{4}{*}{ rs2013208‡ } & RBM5 & HDL (-) & $\mathrm{TT}$ & 39 & 0.22 (0 to 0.73$)$ \\
\hline & & & $\mathrm{TC}$ & 89 & $+0.06(-0.03$ to 0.15$)$ \\
\hline & & & $\mathrm{CC}$ & 56 & $+0.11(0.02$ to 0.21$)$ \\
\hline & & & & & $\mathrm{p}_{\text {trend }}=0.022$ \\
\hline \multirow[t]{4}{*}{ rs9488822 } & FRK & $\mathrm{TC}(+)$ & AA & 77 & $0.24(-0.31$ to 0.89$)$ \\
\hline & & $\operatorname{LDL}(+)$ & AT & 77 & $+0.08(0.00$ to 0.15$)$ \\
\hline & & & TT & 30 & $+0.13(0.03$ to 0.23$)$ \\
\hline & & & & & $\mathrm{p}_{\text {trend }}=0.006$ \\
\hline \multirow[t]{3}{*}{ rs17173637¥ } & TMEM176A & HDL (+) & TC & 23 & $0.19(-0.31$ to 0.57$)$ \\
\hline & & & $\mathrm{TT}$ & 161 & $+0.12(0.01$ to 0.22$)$ \\
\hline & & & & & $p_{\text {trend }}=0.032$ \\
\hline \multirow[t]{3}{*}{ rs10401969 } & CILP2 & $\mathrm{TC}(+)$ & TC & 26 & $0.19(-0.31$ to 0.44$)$ \\
\hline & & Triglycerides (+) & $\mathrm{TT}$ & 158 & $+0.11(0.01$ to 0.21$)$ \\
\hline & & $\operatorname{LDL}(+)$ & & & $p_{\text {trend }}=0.032$ \\
\hline \multirow[t]{4}{*}{ rs2277862 } & ERGIC3 & TC (-) & $\mathrm{CC}$ & 140 & $0.27(-0.31$ to 1.04$)$ \\
\hline & & & $\mathrm{TC}$ & 36 & $+0.05(-0.04$ to 0.14$)$ \\
\hline & & & TT & 8 & $+0.17(-0.00$ to 0.34$)$ \\
\hline & & & & & $\mathrm{p}_{\text {trend }}=0.039$ \\
\hline \multicolumn{6}{|c|}{ Cumulative effects of the five SNPs associated with annualised change in EDSS } \\
\hline$\leq 3$ risk alleles & & & & 73 & $0.21(-0.32$ to 0.89$)$ \\
\hline 4 risk alleles & & & & 47 & $+0.08(-0.00$ to 0.16$)$ \\
\hline 5 risk alleles & & & & 50 & $+0.10(0.02$ to 0.18$)$ \\
\hline \multirow[t]{2}{*}{$\geq 6$ risk alleles } & & & & 14 & $+0.38(0.25$ to 0.51$)$ \\
\hline & & & & & $p_{\text {trend }}=1.4 \times 10^{-6}$ \\
\hline
\end{tabular}

${ }^{*}(-)$ decrease the level of corresponding lipid profile(s); (+) increase the level of corresponding lipid profile(s).

†Adjusted for age, sex, study site and relapse at the fifth-year EDSS assessment.

${ }^{\ddagger} \neq$ For consistency of the directional effect in annualised change in EDSS, the minor allele was set as reference, and the major allele is the risk allele.

${ }^{\S}$ Disability was presented as mean $\triangle E D S S$ for the reference group, and the coefficients relative to reference $(\beta(95 \% \mathrm{CI}))$ are presented for subsequent levels.

EDSS, Expanded Disability Status Scale; GWAS, genome-wide association study; HDL, high-density lipoprotein; LDL, low-density lipoprotein; SNP, single-nucleotide polymorphism; TC, total cholesterol. 
still nominally significant after simulating 50000 times (online supplementary table 4).

When we examined the combined effect on disability level of these five lipid-related SNPs by creating a CGRS, a strong and highly significant dose dependency was seen $\left(\mathrm{p}_{\text {trend }}=1.4 \times 10^{-6}\right)$. The annualised disability progression in the 73 participants who carried $\leq 3$ risk alleles was 0.21 EDSS points per year, while for those who carried $\geq 6$ risk genotypes annual $\triangle$ EDSS was 0.38 points higher. The CGRS model explained $16 \%$ of the variance in $\triangle$ EDSS, after adjustment for age, sex, study site and relapse at the 5-year EDSS assessment. There were some inconsistencies of the $\triangle$ EDSS-associated SNPs in predicting the direction of effect of the corresponding lipid profiles, with only three of the five SNPs acting in the putative biologically expected direction. For example, SNP rs9488822 increased TC levels and increased EDSS progression which is consistent in direction, whereas SNP rs2277862 decreased TC and increased EDSS progression which is inconsistent in direction. We therefore undertook a sensitivity analysis where we included only the three biologically consistent SNPs rs2013208, rs9488822 and rs10401969 genotypes in an abbreviated CGRS. In the sensitivity analysis where we included only SNPs rs2013208, rs9488822 and rs10401969 genotypes in the abbreviated CGRS, this model predicted $9 \%$ of the variance in disability $\left(\mathrm{p}_{\text {trend }}=6.5 \times 10^{-5}\right)$.

\section{Interactions between lipid CGRS and lipid variables in predicting $\triangle E D S S$}

We found a significant interaction between the level of HDL and the TC:HDL ratio and the lipid CGRS in predicting $\triangle$ EDSS that persisted after adjusting for age, sex, study site and relapse at the 5 -year EDSS assessment $\left(\mathrm{p}_{\text {interaction }}\right.$ for $\mathrm{HDL}=0.005, \mathrm{p}_{\text {interaction }}$ for TC:HDL $=0.030)$. There was no association between the level of HDL and the TC:HDL ratio and the lipid CGRS $(\beta=0.15$, $95 \% \mathrm{CI}-0.24$ to $0.53, \mathrm{p}=0.449$ for HDL; $\beta=-0.09,95 \%$ $\mathrm{CI}-0.47$ to $0.29, \mathrm{p}=0.641$ for $\mathrm{TC}: \mathrm{HDL}$ ratio). The $\Delta \mathrm{EDSS}$ was not significantly different among participants who had $\leq 4$ risk alleles, regardless of the level of HDL and TC:HDL ratio (figure 1). However, for participants having more than four risk alleles, a significantly higher $\triangle$ EDSS was observed when they had lower level of HDL or a higher TC:HDL ratio.

The $\mathrm{R}^{2}$ of the model for HDL and EDSS progression, adjusting for age, sex, study site and relapse at the 5 -year review, was 0.04 , but including the lipid CGRS variables increased this to 0.26 . For TC:HDL ratio, the $\mathrm{R}^{2}$ of the model for TC:HDL ratio was
0.01, increasing to 0.27 on including the lipid CGRS. These results demonstrate the significant gene-environment interaction between lipid levels and CGRS in predicting disability progression.

There were small but significant changes in the level of HDL, LDL, non-HDL and TC:HDL ratio over the 5 years with a worsening trend of the lipid variables (data not shown). However, there was no association between the changes in these lipid variables and $\triangle E D S S$, and no interaction was seen between the changes in these lipid variables and the lipid CGRS in predicting $\Delta$ EDSS.

\section{Association between BMI-related SNPs and $\triangle$ EDSS}

One of the 97 BMI-related SNPs, rs2033529, was nominally associated with $\triangle$ EDSS $(\beta=-0.07 ; 95 \%$ CI -0.13 to -0.02 ; $\mathrm{p}=0.012$ ). The permutation simulation analysis for rs2033529 showed that the permutation $\mathrm{p}$ value was still nominally significant $(p=0.014$, online supplementary table 4$)$.

In our dataset, there was no significant change in BMI level over the 5 years. The minor allele of rs2033529 (G) was associated with a lower baseline BMI, although it did not reach significance $(p=0.073)$. Moreover, no significant interaction was found between rs2033529 and baseline BMI in predicting $\Delta$ EDSS $\left(\mathrm{p}_{\text {interaction }}=0.701\right)$.

\section{DISCUSSION}

Using a large prospective study of clinical disease progression in early MS, we showed that five lipid-related SNPs nominally predicted disability progression alone and when combined into a CGRS. The significant interaction between the CGRS generated from the five lipid-related SNPs and HDL and TC:HDL ratio in predicting $\triangle$ EDSS indicated that disability progression in MS was significantly driven by both lipids and lipid-related polymorphisms, with the association between serum lipid levels and $\triangle$ EDSS being stronger for those having more lipid-related risk alleles. No such associations were seen for BMI-related polymorphisms, however.

To our knowledge, this is the first study demonstrating the effect of lipid-related genetic polymorphisms on disability change in MS after controlling for appropriate confounders. Due to the limited sample size and the minimal contribution of each individual susceptibility variant, none of these nominally significant SNP individually remained significant after stringent correction

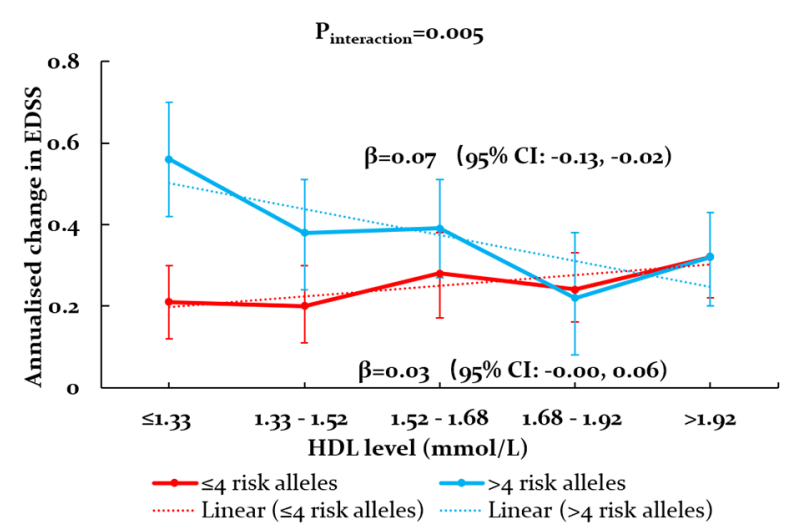

(A)

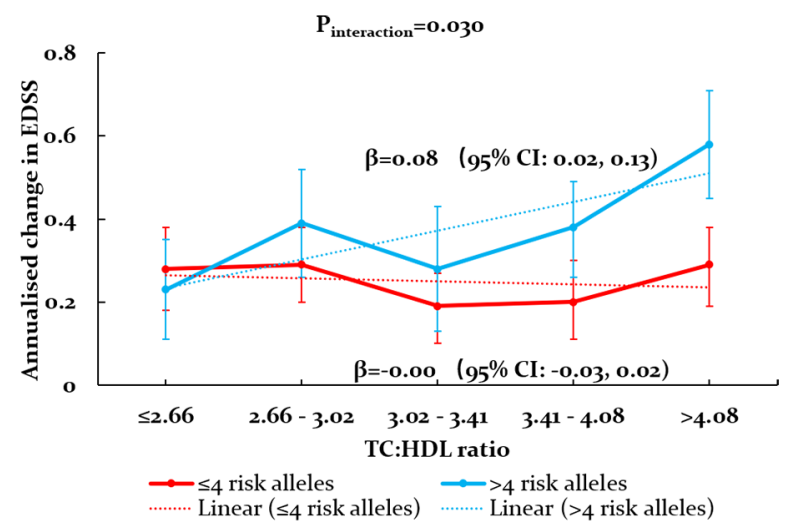

(B)

Figure 1 Predicted mean values of annualised change in Expanded Disability Status Scale (EDSS) with 95\% Cl for different levels of high-density lipoprotein (HDL) (A) and total cholesterol (TC):HDL ratio (B) among participants having $\leq 4$ risk alleles (red line) and those having $>4$ risk alleles (blue line). 
for multiple testing. However, we used other epidemiological supports such as allele dose dependency, strong magnitude of effect and dose dependency of the CGRS analysis to support the validity of the observed associations. The CGRS generated from the five lipid-related SNPs explained 16\% of the variance in MS disability progression, suggesting that these common risk variants in combination significantly contribute to disability progression in early MS. Participants having more risk alleles had a significant positive association with higher $\triangle$ EDSS compared with those having less: for example, the annual change in EDSS for those who had $\geq 6$ risk alleles was 0.38 EDSS points greater per year than those who had $\leq 3$ alleles.

When examining the interaction between lipid levels and the CGRS, we found that the combination of the CGRS and the lipid variable explained $26 \%$ and $27 \%$ of the variance in MS disability progression for HDL and TC:HDL ratio, respectively. This is significantly more than would be expected from the individual effects, suggesting a strong gene-environment interaction. This, along with the observation that the lipid CGRS modulated the association between serum lipid profile and $\triangle$ EDSS, supports the joint role of genetic and lifestyle factors in MS progression and also strongly supports the validity of this methodology.

As noted in the results, among the five lipid-related SNPs, rs17173637 and rs2277862 had conflicting effects on $\triangle$ EDSS where their effects on the corresponding lipid profiles would predict the opposite effect on $\triangle$ EDSS. There are three possible explanations for this. First, no SNP reached significance based on the strict multiple testing criteria; therefore, it may represent type I error and, therefore, these conflicting associations with EDSS are simply spurious. Second, considering the large effect of the CGRS on disability change, it may reflect the complex interaction between SNPs in predicting outcomes, ${ }^{21}$ where the combination of the five lipid-related SNPs synergistically affects the risk of disability progression. Third, the effect of the SNPs may be pleiotropic and thus not just reliant on their effects on specific lipid parameters. ${ }^{22}$ That is, the five lipid-related SNPs along with environmental factors produced a synergistic effect on $\triangle E D S S$, rather than a simple linear pathway. Regardless, when restricting to the three SNPs with a consistent direction of effect on lipid profiles and $\triangle$ EDSS, the CGRS was still strongly significant and still explained 9\% of the variance in $\triangle$ EDSS.

A major strength of this study is the long follow-up time, allowing for clinically meaningful disability change. Stable disability progression and measurement has been documented at 4 years' disease duration, so our 5 -year longitudinal assessment of EDSS should allow reliable measurement of disability change. ${ }^{23}$ Furthermore, potential environmental confounding factors were measured before disability change, and along with the inherited nature of genetic variation, reverse causality should not be an issue. The major limitation of our study is the sample size. Although a sample size of 184 participants is reasonable when considering the long follow-up and restriction to only those who had definite MS, it is difficult to reach stringent measures of statistical significance, the fairly conservative multiple testing approach, Bonferroni correction, may increase type II error (false negatives). ${ }^{24}$ Therefore, we used other methods to overcome this shortcoming, including allele dose response, cumulative genetic risk score analyses, permutation analyses and interaction analyses. However, there still remains a risk of both type I and type II error in this analysis and replication in other similar cohorts would significantly strengthen these findings.

These results may have implications for the controversial results of randomised controlled trials investigating statin therapyeither alone or in combination with standard disease-modifying treatment-on clinical outcomes in MS, where statin therapy reduced the rate of disability progression in some studies, ${ }^{25-28}$ but showed no evidence of a protective effect in others. ${ }^{29}{ }^{30} \mathrm{It}$ may be that these therapies are efficacious only in those with a genetic risk profile disposing them to have negative impacts of dyslipidaemia on MS progression, along the lines of that seen for breast cancer and other conditions. ${ }^{31}$ Therefore, people having these risk polymorphisms may obtain benefit in reducing or modulating the rate of disability accumulation through interventions that modulate an adverse lipid profile, including statin therapy.

Many studies have shown that lipids play a crucial role in MS disability progression as noted in the introduction. Moreover, people with MS with vascular comorbidities (where an adverse lipid profile is a significant risk factor) showed more rapid disability progression. ${ }^{7}$ In addition to these effects of lipids on MS progression, lipid profile may play a crucial role in the development of early MS lesions; indeed, some have argued that MS is a disease resulting from a disorder of lipids. ${ }^{32-34}$ Our results suggest that the nominally $\triangle$ EDSS-associated SNPs may be near or within genes that have significant effects on MS disability progression. For example, the SNP rs2013208 showed a strong association with coronary artery disease, ${ }^{12}$ and a correlation between disability progression in MS and increased risk of coronary artery disease has been shown in a case-control study. ${ }^{35}$ Also, rs2013208 is located within an intron of the gene RBM5, which encodes a long non-coding RNA binding protein whose aberrant expression has been implicated as an underlying driver of MS causation. ${ }^{36}$ The SNP rs17173637, located within an intron of TMEM176A, is significantly upregulated in whole blood samples of people with MS. ${ }^{37}$ The SNP rs2033529 may exert its function through its nearest gene, LRFN2, which is one of the most important genes involved in regeneration of neuronal processes in MS. ${ }^{38}$ However, whether these SNPs influence disability progression through lipids/BMI levels or by interacting with other established MS genetic risk loci similar to their interactions in MS onset ${ }^{39}$ need to be further investigated in functional studies.

In conclusion, our study supports a role for genetic variants that modulate lipid profiles in MS disability progression. By using the aggregation of multiple lipid-related SNPs into a CGRS, we found that the CGRS was significantly associated with increased disability progression in early MS, and the model of the combination of the lipid variable and the CGRS explained more than one quarter of the variation in disability progression in this cohort. The interaction between the CGRS and lipids in predicting disability progression provides further genetic evidence for a link between lipids and MS disability progression where both environmental and genetic factors influence the rate of change in disability. Validation in other cohorts and investigations into the molecular mechanisms and thus potential therapeutic targets would be the appropriate next step.

Acknowledgements We express our heartfelt thanks to the doctors who notified cases to the Ausimmune Study and the participants in the Ausimmune and AusLong studies for their time and energy, without which we could not have realised this work.

Collaborators These authors belong to the Ausimmune/AusLong Investigators Group, and their individual names are not included in the author list:Keith Dear (University of Adelaide, Australia); Terry Dwyer (Oxford Martin School, University of Oxford, England); Leigh Blizzard (Menzies Institute for Medical Research, University of Tasmania, Hobart, Australia); Simon Broadley (School of Medicine, GriffithUniversity, Gold Coast Campus, Australia); Trevor Kilpatrick (Centre for Neurosciences,Department of Anatomy and Neuroscience, University of Melbourne, Melbourne,Australia); David Williamsand Jeanette Lechner-Scott (University of 
Newcastle, Newcastle,Australia); Cameron Shawand Caron Chapman (Barwon Health, Geelong, Australia); Alan Coulthard(University of Queensland, Brisbane, Australia); Michael P Pender (The University of Queensland,Brisbane, Australia) and PatriciaValery (QIMR Berghofer Medical Research Institute, Brisbane, Australia).

Contributors BVT, YaZ, YuZ, JC and IAFvdM were involved in the study concept and design. BVT, RML, IAFvdM, A-LP and KK were involved in data acquisition. YaZ, YuZ, SS and PT were involved in data analysis. SS, YaZ, BVT and YuZ were involved in drafting the manuscript. All authors participated in critical revision of the manuscript.

Funding The AusLong Study was funded by the Australian National Health and Medical Research Council (NHMRC APP544922).

Competing interests A-LP received personal fees from Biogen Pty Ltd and the fees were used for travel and conference presentation. BVT has received travel grants and honoraria for presentations and Advisory Board membership from Biogen Pty Ltd, Merck Serona Ltd, Roche Pty Ltd Novartis Pty Ltd and Sanofi Pty Ltd.

\section{Patient consent for publication Obtained.}

Ethics approval Nine regional human research ethics committees approved the studies.

Provenance and peer review Not commissioned; externally peer reviewed.

\section{REFERENCES}

1 Beecham AH, Patsopoulos NA, Xifara DK, et al. Analysis of immune-related loci identifies 48 new susceptibility variants for multiple sclerosis. Nat Genet 2013;45:1353-60

2 van der Mei IAFet al. Past exposure to sun, skin phenotype, and risk of multiple sclerosis: case-control study. BMJ 2003;327:316.

3 Tettey P, Simpson S, Taylor B, et al. An adverse lipid profile is associated with disability and progression in disability, in people with MS. Mult Scler 2014;20:1737-44.

4 Tettey P, Simpson S, Taylor B, et al. An adverse lipid profile and increased levels of adiposity significantly predict clinical course after a first demyelinating event. J Neurol Neurosurg Psychiatry 2017;88:395-401.

5 Weinstock-Guttman B, Zivadinov R, Mahfooz N, et al. Serum lipid profiles are associated with disability and MRI outcomes in multiple sclerosis. J Neuroinflammation 2011;8:127.

6 Jokubaitis VG, Zhou Y, Butzkueven H, et al. Genotype and phenotype in multiple sclerosis-potential for disease course prediction? Curr Treat Options Neurol 2018;20:1-14.

7 Marrie RA, Rudick R, Horwitz R, et al. Vascular comorbidity is associated with more rapid disability progression in multiple sclerosis. Neurology 2010;74:1041-7.

8 Weinstock-Guttman B, Zivadinov R, Ramanathan M. Inter-dependence of vitamin D levels with serum lipid profiles in multiple sclerosis. J Neurol Sci 2011;311(1 2):86-91.

9 Palavra F, Marado D, Mascarenhas-Melo F, et al. New markers of early cardiovascular risk in multiple sclerosis patients: oxidized-LDL correlates with clinical staging. Dis Markers 2013:34:341-8.

10 Pilutti LA, McAuley E, Motl RW. Weight status and disability in multiple sclerosis: an examination of bi-directional associations over a 24-month period. Mult Scler Relat Disord 2012;1:139-44

11 Marrie R, Horwitz R, Cutter G, et al. High frequency of adverse health behaviors in multiple sclerosis. Mult Scler 2009;15:105-13.

12 Willer CJ, Schmidt EM, Sengupta S, et al. Discovery and refinement of loci associated with lipid levels. Nat Genet 2013;45:1274-83.

13 Proust C, Empana JP, Boutouyrie $\mathrm{P}_{\text {, et }}$ al. Contribution of rare and common genetic variants to plasma lipid levels and carotid stiffness and geometry: a substudy of the Paris prospective study 3. Circ Cardiovasc Genet 2015:8:628-36.

14 Keller M, Schleinitz D, Förster J, et al. THOC5: a novel gene involved in HDLcholesterol metabolism. J Lipid Res 2013;54:3170-6.
15 Locke AE, Kahali B, Berndt SI, et al. Genetic studies of body mass index yield new insights for obesity biology. Nature 2015;518:197-206.

16 Ridker PM. Fasting versus nonfasting triglycerides and the prediction of cardiovascular risk: do we need to revisit the oral triglyceride tolerance test? Clin Chem 2008;54:11-13.

17 Zhou Y, Graves JS, Simpson S. Genetic variation in the gene Lrp2 increases relapse risk in multiple sclerosis. J Neurol Neurosurg Psychiatry 2017:jnnp-2017-315971.

18 Holm S. A simple sequentially rejective multiple test procedure. Scandinavian journal of statistics 1979:65-70.

19 Pan G, Simpson S, Mei vander I. Role of genetic susceptibility variants in predicting clinical course in multiple sclerosis: a cohort study. J Neurol Neurosurg Psychiatry 2016:jnnp-2016-313722.

20 Morrison AC, Voorman A, Johnson AD, et al. Whole-genome sequence-based analysis of high-density lipoprotein cholesterol. Nat Genet 2013;45:899-901.

21 Ottman R. Gene-environment interaction: definitions and study designs. Prev Med 1996;25:764-70.

22 Deng Y, Pan W. Testing genetic pleiotropy with GWAS summary statistics for marginal and conditional analyses. Genetics 2017;207:1285-99.

23 Hughes S, Spelman T, Trojano M. The Kurtzke EDSS RANK stability increases 4 years after the onset of multiple sclerosis: results from the MSBase registry. J Neurol Neurosurg Psychiatry 2011:jnnp-2011-301051.

24 Perneger TV. What's wrong with Bonferroni adjustments. BMJ 1998;316:1236-8.

25 Chataway J, Schuerer N, Alsanousi A. The MS-STAT trial: High dose simvastatin slows brain atrophy and delays disability in secondary progressive multiple sclerosis: a phase II placebo-controlled trial (PL02. 001). Neurology 2013;80(Suppl 7):PL02. 001-PL02. 01.

26 Bhardwaj S, Coleman Cl, Sobieraj DM. Efficacy of statins in combination with interferon therapy in multiple sclerosis: a meta-analysis. Am J Health Syst Pharm 2012;69:1494-9.

27 Lanzillo R, Orefice G, Quarantelli M, et al. Atorvastatin combined to interferon to verify the efficacy (active) in relapsing-remitting active multiple sclerosis patients: a longitudinal controlled trial of combination therapy. Mult Scler 2010;16:450-4.

28 Togha M, Karvigh SA, Nabavi M, et al. Simvastatin treatment in patients with relapsing-remitting multiple sclerosis receiving interferon beta $1 \mathrm{~A}$ : a double-blind randomized controlled trial. Mult Scler 2010;16:848-54.

29 Rudick RA, Pace A, Rani MR, et al. Effect of statins on clinical and molecular responses to intramuscular interferon beta-1a. Neurology 2009;72:1989-93.

30 Sorensen PS, Lycke J, Erälinna JP, et al. Simvastatin as add-on therapy to interferon $\beta$-1a for relapsing-remitting multiple sclerosis (SIMCOMBIN study): a placebocontrolled randomised phase 4 trial. Lancet Neurol 2011;10:691-701.

31 Mangravite LM, Wilke RA, Zhang J, et al. Pharmacogenomics of statin response. Curr Opin Mol Ther 2008;10:555-61.

32 Weinstock-Guttman B, Zivadinov R, Horakova D, et al. Lipid profiles are associated with lesion formation over 24 months in interferon- $\beta$ treated patients following the first demyelinating event. J Neurol Neurosurg Psychiatry 2013;84:1186-91.

33 Newcombe J, Li H, Cuzner ML. Low density lipoprotein uptake by macrophages in multiple sclerosis plaques: implications for pathogenesis. Neuropathol App/ Neurobiol 1994:20:152-62

34 Corthals AP. Multiple sclerosis is not a disease of the immune system. Q Rev Biol 2011;86:287-321.

35 Moccia M, Lanzillo R, Palladino R, et al. The Framingham cardiovascular risk score in multiple sclerosis. Eur J Neurol 2015;22:1176-83.

36 Eftekharian MM, Ghafouri-Fard S, Soudyab M, et al. Expression analysis of long non-coding RNAs in the blood of multiple sclerosis patients. J Mol Neurosci 2017:63:333-41.

37 Nickles D, Chen HP, Li MM, et al. Blood RNA profiling in a large cohort of multiple sclerosis patients and healthy controls. Hum Mol Genet 2013:22:4194-205.

38 Fischer MT, Wimmer I, Höftberger R, et al. Disease-specific molecular events in cortical multiple sclerosis lesions. Brain 2013;136(Pt 6):1799-815.

39 Gianfrancesco MA, Barcellos LF. Obesity and multiple sclerosis susceptibility: a review. J Neurol Neuromedicine 2016:1:1. 